\title{
Profile of Childhood Epilepsies and Syndromes Seen in an Electrophysiology Laboratory in Southeast Nigeria
}

\author{
Christian Chukwukere Ogoke $e^{\mathrm{a}, \mathrm{e}}$, Wilson Chukwuneke Igwe ${ }^{\mathrm{b}}$, \\ Ifeanyi Innocent Ike ${ }^{\mathrm{c}}$, Edmund Ndudi Ossai ${ }^{\mathrm{d}}$
}

\begin{abstract}
Background: Epilepsy and other seizure disorders are highly prevalent in resource-poor countries. Electroencephalogram (EEG) plays an invaluable role in differentiating seizure types, epilepsy syndromes and choice of appropriate anti-epileptic drugs. This is critical for effective management of epilepsies including their prognostication. The profile of childhood epilepsies in our locality is unknown. This study was therefore carried out to ascertain the prevalent electro-clinical syndromes and epilepsy types, etiological factors and co-morbid conditions in children who had EEG in Owerri, Southeast Nigeria.

Methods: The clinical data including the Basic Data Sheet and EEG records of all children with epilepsy seen between January 2017 and December 2018 at the EEG Unit/Child Neurology Clinic of Mother Healthcare Diagnostics \& Hospital, Owerri were retrospectively reviewed. Relevant data from each patient's clinical history, physical examination, investigation findings and EEG diagnosis of seizure type and epilepsy syndrome were extracted and recorded.

Results: Out of 97 children with epilepsy, 57 were males and 40 were females giving an male/female $(\mathrm{M} / \mathrm{F})$ ratio of 1.4:1. Focal epilepsy (31\%) was the most frequent epilepsy type, while benign rolandic epilepsy (BRE) (14\%) was the most common epilepsy syndrome seen. West syndrome was the second most prevalent epilepsy syndrome. Epilepsy was of genetic etiology in 48 (50\%) and 22 (23\%) had a history of severe perinatal asphyxia. No co-morbidity was detected in 70 $(72 \%)$ patients but among those with co-morbidity, communication disorder (12.4\%) was the most frequent.
\end{abstract}

Conclusions: Focal epilepsies and syndromes of genetic etiology

Manuscript submitted August 13, 2019, accepted August 23, 2019

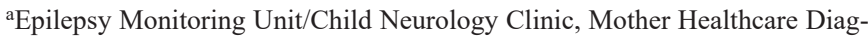
nostics \& Hospital, 5B Okigwe Road, Owerri, Nigeria

bDepartment of Paediatrics, Nnamdi Azikiwe University, Nnewi Campus \& Nnamdi Azikiwe University Teaching Hospital, Nnewi, Anambra State, Nigeria

'Department of Paediatrics, Federal Medical Centre, Owerri, Nigeria

dDepartment of Community Medicine, College of Health Sciences, Ebony State University, Abakiliki, Nigeria

${ }^{\mathrm{e} C o r r e s p o n d i n g ~ A u t h o r: ~ C h r i s t i a n ~ O g o k e, ~ M o n i t o r i n g ~ U n i t / C h i l d ~ N e u r o l o g y ~}$ Clinic, Mother Healthcare Diagnostics \& Hospital, 5B Okigwe Road, Owerri,

Nigeria. Email: chrischikere@yahoo.com

doi: https://doi.org/10.14740/ijcp344 were the more prevalent epilepsy types and syndromes in our locality. Majority of the children had no co-morbid conditions but most of those with co-morbidity would require speech therapy. Epileptic encephalopathies were also frequent and this underscores the need to prevent severe perinatal asphyxia and central nervous system (CNS) infections.

Keywords: Childhood epilepsies; Syndromes; Etiology; Co-morbidities

\section{Introduction}

Epilepsy remains the commonest neurological disorder seen in the Paediatric Neurology Clinics of most resource constrained countries including Nigeria [1-4]. It is an important cause of morbidity and mortality in these areas. Moreover, the cost of treatment which is usually borne by the parents is extremely high and out of reach of many households in Nigeria [5].

Currently, epilepsy is defined as a disease of the brain manifested clinically by two or more unprovoked (or reflex) seizures occurring $>24 \mathrm{~h}$ apart or one unprovoked (or reflex) seizure with a probability of $\geq 60 \%$ of having further seizures over the next 10 years or a diagnosis of an epilepsy syndrome [6]. Epilepsy syndromes refer to clusters of features (seizure type(s), electroencephalogram (EEG) findings, imaging findings, age-dependent features, triggers and sometimes prognosis) that occur together [6]. The classification of epilepsy types and syndromes provides adequate information about patients with epilepsy and informs proper management including prognostication [6]. There are many epilepsy syndromes and EEG plays an invaluable role as a supportive evidence for the diagnosis and differentiation of epilepsies and syndromes.

There are only few centers in Nigeria with neurodiagnostic tests in addition to a dearth of pediatric neurologists resulting in a "diagnostic gap". This makes management of epilepsy in Nigeria a challenging task [7, 8]. Recently, digital pediatric EEG recording and neuroimaging studies have become available in Owerri and their application has been encouraging. Therefore, this study was carried out to ascertain the common electro-clinical syndromes and epilepsy types, etiological factors and co-occurring conditions in children who had EEG in a referral center in Owerri, Southeast Nigeria. 


\section{Materials and Methods}

The study was carried out at the Electrophysiology Laboratory/Child Neurology Clinic of Mother Healthcare Diagnostics \& Hospital, Owerri. Mother Healthcare Diagnostics \& Hospital is a multi-specialist hospital and diagnostic center with an EEG Unit/Child Neurology Clinic that was the first to commence pediatric digital EEG recording in Owerri, Southeast Nigeria. This was a retrospective study of all children with epilepsy who were seen at the facility over a period of 2 years. This research was approved by the Federal Medical Centre Owerri Health Research Ethics Committee (approval number: FMC/OW/HREC/232) before commencement of the study. This study was conducted in compliance with the ethical standards of the responsible institution on human subjects as well as with the Helsinki Declaration.

\section{Study population}

The cohort consisted of children aged $<18$ years, with a median age of 5 years (range 0.33 - 17.67 years), who were seen at the facility between January 2017 and December 2018. Children who had febrile seizures, normal EEG after single unprovoked seizures, or those whose clinical and EEG findings supported nonepileptic paroxysmal events (NEPEs) were excluded from the study. Neonates were not seen during the period under review.

\section{Study protocol}

Each patient's clinical history, physical examination findings, basic data sheet for EEG, EEG findings and other investigation results including neuroimaging of the brain, if done, were reviewed and relevant data were recorded on a pro forma. Each patient's clinical history and EEG findings were used to classify their seizure types into: focal, generalized, combined generalized and focal and unknown onset. Diagnosis and classification of epilepsy types based on the etiology, EEG findings and semiology, and epilepsy syndrome were done according to the current 2017 International League Against Epilepsy (ILAE) recommendations $[6,9]$. The patients were categorized into focal, generalized or combined seizures types if they had both focal and generalized seizures. Those with the features specific for an epilepsy syndrome including syndrome-specific EEG abnormalities were classified accordingly. Those with all clinical features of a syndrome but with a normal EEG were classified according to their seizure type(s) only. It was only in the cases of Watanabe-Vigevano syndrome that a normal inter-ictal EEG contributed to the diagnosis of the syndrome. It is necessary to mention that those children with typical clinical features of a syndrome and a normal first EEG could have manifested EEGspecific abnormalities on subsequent EEGs. However, all patients in this study had only a first EEG study.

In the etiologic classification in this study, a structural etiology was accepted based on abnormal neuroimaging findings of computed tomography (CT) scan or magnetic resonance imaging (MRI). Those who had a probable structural cause for their
Table 1. Age Distribution of Study Population

\begin{tabular}{lll}
\hline Age of respondents (groups) & Frequency $(\mathbf{n}=\mathbf{9 7})$ & Percent $(\mathbf{\%})$ \\
\hline$<1$ year & 11 & 11.3 \\
$1-<5$ years & 36 & 37.1 \\
$5-<10$ years & 30 & 30.9 \\
$10-<15$ years & 17 & 17.5 \\
$15-<18$ years & 3 & 3.1 \\
\hline
\end{tabular}

epilepsy such as head injury with impact seizures, focal seizures and focal background slowing on EEG but had no brain imaging (CT or MRI) were categorized as unclear/multiple (unknown). This is because some also had features suggestive of other etiologic categories such as a positive family history, thereby making it difficult to pin the etiology to one category since a structural cause was not proven. Thus, a good percentage of patients with post traumatic epilepsy fell under the unknown category. Patients categorized as having a genetic etiology had a relevant family history, typical seizure semiology, normal pregnancy, birth history, normal development, normal neurological examination and normal EEG background. Those classified as having an infectious etiology were neurologically normal prior to the onset of the central nervous system infections.

Children with a history suggestive of severe birth asphyxia (prolonged/obstructed labor, not crying immediately after birth, with or without post asphyxia seizures within $24-48$ $\mathrm{h}$ post delivery) without any neuroimaging studies were categorized as unknown since the cortical injury due to asphyxia was not proven. However some of these had microcephaly or speech/language disorder that suggested cortical injury.

\section{Data analysis}

Data were entered into a computer and analyzed using IBM SPSS statistical software version 25. Descriptive statistics such as frequencies and percentages, mean, range and standard deviation (SD) were calculated.

\section{Results}

Out of the 115 children who had a first EEG, 97 had epilepsy, giving a prevalence of $83 \% ; 57(58.8 \%)$ were males and 40 $(41.2 \%)$ were females giving a male/female $(\mathrm{M} / \mathrm{F})$ gender ratio of 1.43:1. Their ages ranged $0.33-17.67$ years with a mean \pm $\mathrm{SD}$ of $5.7 \pm 4.4$ years. The age distribution of the study population is shown in Table 1.

A summary of the salient clinical features seen in the study population is shown in Table 2.

EEG was normal in $33(34 \%)$ of the children, abnormal in $52(53.6 \%)$ and were not interpretable in $12(12.45)$ because of poor quality. EEG contributed to epilepsy syndrome diagnosis in $38(39.2 \%)$ of cases. Focal epilepsy (30.9\%) was the most frequent epilepsy type, while benign rolandic epilepsy (BRE) $(14.4 \%)$ was the most common epilepsy syndrome (Table 3 ). 
Table 2. Salient Clinical Features in the Study Population

\begin{tabular}{|lll}
\hline Clinical/physical finding* & $\begin{array}{l}\text { Frequency } \\
(\mathbf{n = 9 7 )}\end{array}$ & $\begin{array}{l}\text { Percent } \\
(\mathbf{\%})\end{array}$ \\
\hline Family history of seizures & 65 & 67 \\
\hline Anti-epileptic drug therapy & 46 & 47.4 \\
Co-morbidity (at least once) & 27 & 27.8 \\
\hline Developmental delay & 25 & 25.8 \\
Severe birth asphyxia & 22 & 22.7 \\
Microcephaly & 14 & 14.4 \\
Neuroimaging (CT/MRI) & 12 & 12.4 \\
Neonatal seizures & 11 & 11.3 \\
Poor school performance & 11 & 11.3 \\
Previous history of meningitis & 7 & 7.2 \\
Developmental regression & 5 & 5.2 \\
Macrocephaly & 5 & 5.2 \\
Preterm delivery & 4 & 4.1 \\
\hline Head trauma with impact seizures & 4 & 4.1 \\
\hline Post term delivery & 1 & 1.0 \\
\hline
\end{tabular}

*Multiple responses encouraged. CT: computed tomography; MRI: magnetic resonance imaging. 4).

Half of the study population had a genetic etiology (Table

The spectrum of co-morbidities occurring singly or in various combinations include: cerebral palsy, microcephaly, macrocephaly, arrested hydrocephalus, communication disorder, hyperactivity, global developmental delay (GDD), intellectual disability (ID), congenital bilateral cataracts and cerebral visual impairment. There were no co-morbidities in $70(72.2 \%)$ of the study population, $14(14.4 \%)$ had one, eight (8.2\%) had two comorbidities, while five $(5.2 \%)$ had three accompanying impairments. Either singly or in combination with other co-morbidities, $12(12.4 \%)$ of the children had communication disorder, nine $(9.3 \%)$ had microcephaly, eight $(8.2 \%)$ had cerebral palsy
Table 4. Etiology of Epilepsy

\begin{tabular}{lll}
\hline Etiology & Frequency $(\mathbf{n = 9 7 )}$ & Percent $(\%)$ \\
\hline Genetic & 48 & 49.5 \\
Infectious & 1 & 1.0 \\
Structural & 4 & 4.1 \\
Unknown/multiple & 44 & 45.4 \\
\hline
\end{tabular}

and eight $(8.2 \%)$ had intellectual disability, thereby making communication disorder the most frequent co-morbidity seen.

\section{Discussion}

Epilepsy remains the most prevalent chronic neurological disease in children in low-middle income countries (LMIC) like Nigeria [1-4]. Accurate diagnosis of epilepsy type or syndrome is necessary to provide accurate information and thus further classification beyond that of seizure type becomes imperative [6]. The present study has shown that focal epilepsy is the most common childhood epilepsy in our setting. Similar findings have been reported from other parts of Africa $[10,11]$ and Europe $[12,13]$. However, Eyong et al [14] in their study in South Nigeria reported generalized epilepsy as the commonest epilepsy type seen. The perceived difference is most likely due to the incorporation of EEG findings in diagnosis in our study since the study by Eyong et al [14] was based solely on history obtained from parents/caregivers (eye witness account). Additionally, it may be because some of the focal seizures or focal to bilateral tonic-clonic seizures were wrongly classified as generalized seizures. In a study in Uganda by Kaiser et al [15], the predominant seizure type clinically diagnosed as generalized seizures $(63 \%)$ fell to $22 \%$, while focal seizures increased from $24 \%$ to $78 \%$ after inclusion of EEG findings. So, it is likely that with repeat or further EEGs in this group, the proportion of focal epilepsy and focal epilepsy syndromes will increase. Nevertheless, an earlier study in Southwest Nigeria

Table 3. Spectrum of Epilepsy Types and Syndromes in the Patients

\begin{tabular}{lll}
\hline Epilepsy type/syndrome & Frequency (n=97) & Percent (\%) \\
\hline Focal epilepsy & 30 & 30.9 \\
Generalized epilepsy & 20 & 20.6 \\
Benign rolandic epilepsy (BRE) & 14 & 14.4 \\
West syndrome & 11 & 11.3 \\
Combined generalized and focal epilepsy & 4 & 4.1 \\
Panayiotopoulos syndrome (PS) & 4 & 4.1 \\
Childhood absence epilepsy (CAE) & 3 & 3.1 \\
Watanabe-Vigevano syndrome & 2 & 2.1 \\
Generalized epilepsy with febrile seizure plus (GEFS+) & 1 & 1.0 \\
Idiopathic generalized epilepsy with GTCSs only & 1 & 1.0 \\
Juvenile myoclonic epilepsy (Janz syndrome) & 1 & 1.0 \\
Lennox Gastaut syndrome (LGS) & 1 & 1.0
\end{tabular}


by Lagunju et al [16] reported a predominance of generalized epilepsies (67.8\%). This difference between this present study and earlier studies in Nigeria is difficult to explain and further studies are needed for clarification since only the present study which is from a different geo-political region from earlier studies in Nigeria has findings that agree with international reports.

EEGs play an invaluable role in making accurate diagnosis of seizure type and epilepsy syndrome which guides management and informs prognosis [17]. In this study, EEG contributed to an epilepsy syndrome diagnosis in $39.2 \%$ of cases which is similar to findings by Larsson and Eeg-Oloffson [12] in Sweden. However, Ackermann et al [11] in South Africa reported slightly lower proportions of epilepsy syndromes $(25 \%)$. This observed difference may be due to the larger sample size in their study. The most common epilepsy syndrome reported in this study was BRE and agrees with the findings of Kandil et al [10] in Egypt and Larsson and Eeg-Olofsson [12] in Sweden. However, the reported prevalence of BRE (14.4\%) in this study is lower than that (22\%) reported by Freitag et al [13] in Germany. The children in our study had only a first routine EEG and this may explain the lower prevalence since with repeat EEGs or sleep-deprived EEGs some children with BRE who had an initial normal record will show centro-temporal spikes. The study by Freitag et al [13] recorded awake and or sleep-deprived EEG in all cases. BRE is the commonest childhood epilepsy syndrome and accurate and early diagnosis helps in the choice of appropriate anti-epileptic drug therapy and prognosis.

West syndrome is a serious age-related specific epileptic encephalopathy with multiple and diverse etiologies characterized by epileptic (infantile) spasms, hypsarrhythmia pattern in EEG, and progressive cognitive and neuropsychological deterioration [18]. It was the second commonest epilepsy syndrome in the present study and portends poor neurodevelopmental and cognitive outcome for one out of every 10 children referred for EEG in our facility. An EEG in the untreated state is most likely to be abnormal in West syndrome and other epileptic encephalopathies and may explain their relatively high prevalence in this present study where the patients had only a first EEG. The prevalence of West syndrome implies that there is need to address preventable etiological factors for West syndrome such as severe birth asphyxia and meningitis since a past history of these was present in $22.7 \%$ and $7.2 \%$ of the children, respectively. This finding also means that children with West syndrome contributed significantly to those with developmental delay or regression that was present in one out of every three patients in the cohort. Compared to the study by Freitag et al [13] in Germany that did not find any case of West syndrome or Lennox Gastaut syndrome, this present study reported a relatively high proportion of epileptic encephalopathies. Therefore, this finding agrees with that of Lagunju et al [16] that noted a large burden of symptomatic epilepsy in Nigerian children. However, this was not reflected in the etiology of epilepsy reported in our study (4\% structural and $1 \%$ infectious). This may be because most of the children with epileptic encephalopathies were not fully investigated or had not been fully investigated at the time of assessment and hence being grouped as of unknown/multiple (45\%) etiologies.

The majority $(67 \%)$ of patients in this present study had a positive family history of epileptic seizures and is reflected in almost half of them having a genetic etiology for their epilepsy. This is comparable to the findings by Freitag et al [13]. None of our patients had any molecular genetic studies but according to the ILAE Task Force, having a relevant family history and typical features (seizure semiology and EEG) without molecular genetics is sufficient for a genetic etiologic classification [6]. Patients categorized as having a genetic etiology in this present study all had a relevant family history, typical seizure semiology, normal pregnancy and delivery without any history suggestive of severe birth asphyxia, normal development, normal neurological examination and normal EEG background with or without typical epileptiform abnormalities. Therefore, the profile of epilepsy syndromes diagnosed in this study is in agreement with the predominant genetic etiology since seven out of the nine epilepsy syndromes are genetic epilepsies.

The unavailability, inaccessibility and unaffordability of neurodiagnostic tests in developing countries frequently results in a "diagnostic gap in these settings" [7]. A significant proportion $(45 \%)$ of the study population had an unknown etiology for their epilepsies due to inadequate investigations. Moreso, no metabolic epilepsies or immune etiologies were reported in this study due to diagnostic limitations in our resource-poor setting. Many of the patients in the unknown/multiple category were those that had multiple etiological factors who were not sufficiently investigated to elucidate the etiology. Furthermore, only $12 \%$ of the patients had neuroimaging (CT/MRI of brain) studies unlike the study in Germany [13], where all patients with suspected symptomatic or probably symptomatic etiology had a brain MRI. Therefore, there is need for provision of adequate investigative facilities and neurodiagnostic equipment to improve identification of epilepsy etiologies owing to its importance in management and prognostication [7].

Epilepsy is thought to be not just a neurological disorder but also a systemic disorder with a systemic dysfunction that explains the high prevalence of co-morbidity [19]. This study reported at least one co-morbidity in $28 \%$ of the patients reviewed and this is lower than $74 \%$ and $47.3 \%$ reported in South Africa [11] and Sweden [12], respectively. The present study reviewed patients' data over a short period while the studies by Ackermann et al [11] in South Africa and Larsson and EegOloffson [12] in Sweden reviewed data over longer periods. Neurologic impairments in children are known to manifest later in life as the brain continues to mature and so it is likely that a higher proportion of the patients in this study may manifest more co-morbid conditions if followed up for years. On the other hand, it is also possible that the relatively high proportion of patients without any co-morbidity in this study may be due to the predominance of genetic etiology or genetic epilepsies/ syndromes since they frequently occur in normally developing children with no structural brain abnormalities. Nevertheless, the implication of the co-morbid disorders in one-quarter of our patients is the need to provide multidisciplinary services for their management especially speech therapy since communication disorder was the most frequent co-morbidity seen.

\section{Limitations}

The study is a cross-sectional retrospective study involving a 
small sample size. Follow-up of the patients, repeat EEGs for those with a normal initial record and treatment outcome of these patients would have provided more data for this study.

\section{Conclusions}

Focal epilepsies and syndromes of genetic etiologies were the more prevalent epilepsy types and syndromes in our locality. Majority of these patients did not have any co-morbidities. Speech impairment was the most common abnormality in those with co-morbidities. Epileptic encephalopathies were also frequent and this underscores the need to prevent severe perinatal asphyxia and CNS infections.

\section{Acknowledgments}

We thank the management and staff of Mother Healthcare Diagnostics \& Hospital, Owerri for their support during the period of data acquisition.

\section{Financial Disclosure}

None to declare.

\section{Conflict of Interest}

None to declare.

\section{Informed Consent}

Not applicable.

\section{Author Contributions}

$\mathrm{CCO}$ conceived and designed the topic, recorded and interpreted all EEGs and drafted the article; WCI was a major contributor in writing the manuscript, he revised the article critically for important intellectual content and gave the final approval of the version to be published; III made substantial contribution to the design of the topic, data acquisition and revision of the article; ENO did the data analysis interpretation of data. All authors read and approved the final manuscript.

\section{References}

1. Lagunju IA, Okafor OO. An analysis of disorders seen at the Paediatric Neurology Clinic, University College Hospital, Ibadan, Nigeria. WAJM. 2009;28(1):328-332.

2. Izuora GI, Iloeje SO. A review of neurological disorders seen at the Paediatric Neurology Clinic of the University of Nigeria Teaching Hospital, Enugu. Ann Trop Paediatr. 1989;9(4):185-190.

3. Frank-Briggs AI, EA DA. Pattern of paediatric neurological disorders in port harcourt, Nigeria. Int J Biomed Sci. 2011;7(2):145-149.

4. Ejeliogu EU, Yiltok ES. Pediatric neurologic disorders at a tertiary healthcare facility in North-central Nigeria: A 5 year review. INDJ. 2017;9(4):1-8.

5. Ughasoro MD, Onwujekwe OE, Ojinnaka NC. Economic cost of treatment of childhood epilepsy in Enugu, southeast Nigeria. Int J Technol Assess Health Care. 2014;30(4):469-474.

6. Falco-Walter JJ, Scheffer IE, Fisher RS. The new definition and classification of seizures and epilepsy. Epilepsy Res. 2018;139:73-79.

7. McLane HC, Berkowitz AL, Patenaude BN, McKenzie ED, Wolper E, Wahlster S, Fink G, et al. Availability, accessibility, and affordability of neurodiagnostic tests in 37 countries. Neurology. 2015;85(18):1614-1622.

8. Igwe WC. Challenges in the management of pediatric epilepsy in Nigeria. Trop J Med Res. 2016;19:1-4.

9. Fisher RS, Cross JH, D'Souza C, French JA, Haut SR, Higurashi N, Hirsch E, et al. Instruction manual for the ILAE 2017 operational classification of seizure types. Epilepsia. 2017;58(4):531-542.

10. Kandil MR, Ahmed WM, Sayed AM, Hamed SA. Pattern of epilepsy in childhood and adolescence: A hospitalbased study. AJNS. 2008;26(1):33-44.

11. Ackermann S, Le Roux S, Wilmshurst JM. Epidemiology of children with epilepsy at a tertiary referral centre in South Africa. Seizure. 2019;70:82-89.

12. Larsson K, Eeg-Olofsson O. A population based study of epilepsy in children from a Swedish county. Eur J Paediatr Neurol. 2006;10(3):107-113.

13. Freitag CM, May TW, Pfafflin M, Konig S, Rating D. Incidence of epilepsies and epileptic syndromes in children and adolescents: a population-based prospective study in Germany. Epilepsia. 2001;42(8):979-985.

14. Eyong KI, Ekanem EE, Asindi AA, Chimaeze T. Clinical profile of childhood epilepsy in Nigerian children seen in a tertiary hospital. Int J Contemp Pediatr. 2017;4(4):11381141.

15. Kaiser C, Benninger C, Asaba G, Mugisa C, Kabagambe G, Kipp W, Rating D. Clinical and electro-clinical classification of epileptic seizure in west Uganda. Bull Soc Pathol Exot. 2000;93(4):255-259.

16. Lagunju IA, Fatunde O, Inyang T. Profile of childhood epilepsies in Nigeria. J Paediatr Neurol. 2009;7(2):135-140.

17. Panayiotopoulos CP. General aspects of epilepsies. In: Panayiotopoulos CP (eds). A clinical guide to epileptic syndromes and their treatment 2 nd ed. London: Springer Healthcare Ltd; 2010:1-19.

18. Panayiotopoulos CP. Epileptic encephalopathies in infancy and early childhood. In: Panayiotopoulos CP (eds). A clinical guide to epileptic syndromes and their treatment 2nd ed. London: Springer Healthcare Ltd; 2010. 275-326.

19. Yuen AWC, Keezer MR, Sander JW. Epilepsy is a neurological and a systemic disorder. Epilepsy Behav. 2018;78:57-61. 